\title{
Les fils textuels des violences familiales au Mali
}

\section{Sébastien Le Potvin}

\section{(2) OpenEdition}

Journals

Édition électronique

URL : http://journals.openedition.org/apad/2953

DOI : 10.4000/apad.2953

ISSN : 1950-6929

Éditeur

LIT Verlag

Édition imprimée

Date de publication : 4 avril 2008

Référence électronique

Sébastien Le Potvin, «Les fils textuels des violences familiales au Mali », Bulletin de l'APAD [En ligne], 27-28 | 2008, mis en ligne le 17 juin 2008, consulté le 07 septembre 2020. URL : http://

journals.openedition.org/apad/2953; DOI : https://doi.org/10.4000/apad.2953

Ce document a été généré automatiquement le 7 septembre 2020

Bulletin de l'APAD 


\title{
Les fils textuels des violences familiales au Mali
}

\author{
Sébastien Le Potvin
}

Notre société a terrorisé les femmes et les jeunes...Un jour les enfants de la rancour se mettront ensemble, et ils mettront le feu à la barbe et aux tresses de leurs pères. (Ismaïla Samba Traoré, Les Amants de l'Esclaverie, 2004)

Toiles de soi

1 Des enfants hilares rouent de coups une vieille femme dénudée sous le regard jubilatoire des habitants : la vieille serait « une mangeuse d'âme ». Ailleurs, une fillette assiste au massacre de sa famille. Ces scènes qui inaugurent les œuvres littéraires de Moussa Konaté (Prix de l'âme) et Aïda Mady Diallo (Kouty, Mémoire de sang) contrastent avec l'image paisible du Mali. A contre-courant des discours publics, les œuvres contemporaines des écrivains maliens se révèlent littéralement violentes, décrivant une société qui, faute de repères identifiés, tâtonne en aveugle vers l'avenir, ou plutôt en faisant la sourde oreille aux questions sociales cruciales qui la concernent. Quelles violences, dissimulées aux regards extérieurs et à la conversation publique, sévissent à l'intérieur des cours d'habitation? La littérature lève quelques voiles. René Girard, commentant l'idée que Platon se faisait de la cité parfaite excluant les poètes, interroge si, au fond, ce n'était pas la capacité des textes à révéler « le mensonge du sacrifice » qui dérangeait le philosophe (Le sacrifice, 2003).

2 Nous avons donc questionné les œuvres littéraires maliennes qui abordent les violences familiales, à partir de leur capacité à dépasser la bienséance de la conversation, les convenances obligées du grand parler (Hampâté Bâ). A partir d'un «oser-dire " qui transgresse le musalaka. En effet, la critique directe par la parole est contraire à la culture malienne. Forme de pudeur ou art du consensus et de l'évitement des conflits, le musalaka se caractérise aussi par l'esquive des sujets qui fâchent: «Tu veux savoir pourquoi je fais ça [la prostitution] ? ...Tu sais, on ne dit pas sa vie à quelqu'un que l'on ne connaît pas / Bien sûr. Même à ceux que l'on connaît d'ailleurs " (Ruchers de la capitale: 95). Sorte de contrôle social de la parole publique, ou « hypocrisie sociale » selon les termes de l'ancien président ${ }^{1}$, le musalaka se traduit concrètement par une absence culturelle du débat public. Conscient que «chacun se sert de tout pour 
s'encourager dans son sens, et précipite tout dans sa preuve » (Gide, Voyage au Congo), et que les œuvres littéraires ne constituent ni des études psychosociales, ni des documents sociologiques, nous ne traiterons pas ici des violences familiales au Mali mais des violences familiales telles qu'elles apparaissent dans les textes maliens. Pourquoi étudier des créations fictionnelles qui s'identifient moins à des tableaux ethnographiques qu'à une radiographie filtrée des états d'âme de leurs auteurs? D'abord, un premier constat: en 2003, Alain Marie rappelait que la violence sociale «a longtemps été euphémisée, sinon oubliée $»^{2}$. Or, les violences sociales se répandent à flots dans toutes les littératures africaines depuis près de trente ans ! Mais qui les lit ?... Ensuite, les œuvres littéraires proposent une observation intérieure qui procède de recréations et d'interprétations certes subjectives mais qui donnent à lire l'impensé actuel des violences familiales. Au-delà du contenu narratif et des effets esthétisants, « la parole tisse ce qui est » (Kane, L'aventure ambiguë). Il faut scruter les arrière-plans, les répétitions des thèmes, des situations ou les expressions récurrentes des dialogues ainsi que la filiation entre les textes (l'intertextualité) qui trament des représentations littéraires communes de la société malienne.

3 L'observation des textes, tissus de signes, permet une attention aux coutures - ou points de suture - où se faufile l'imprévu du créateur, où se glisse l'inconscient de l'auteur pour qui «il s'agit moins de penser la violence à l'aide de la littérature que d'écrire une littérature violente qui sorte la pensée de ses ornières, qui nous force à véritablement penser. $»^{3}$ Aux sciences humaines, même si elles n'échappent pas aux contraintes de la littéralité, de profiter des failles percées par les œuvres littéraires pour tenter de muer une "vérité de paroles» (Bonnefoy) en une approche plus factuelle et objective, en une sorte de vérité sociale, bien que le discernement de ces violences débordent sans cesse de la clôture du discours scientifique. Etre attentif aux discours littéraires, enfin, c'est au minimum être à l'écoute des porte-parole des sans-voix et des porte-voix de la parole libre.

La littérature dite moderne émergea au Mali à la fin des années cinquante avec les publications successives de La Passion de Djimé (1956) de Fily Dabo Sissoko et de Sous l'orage (1957) de Seydou Badian Kouyaté4. Les premières œuvres littéraires répondirent le plus souvent aux critères d'une littérature pédagogique et idéologique en vigueur dans les années 1950-70. Cependant, tandis que la négritude triomphait en grand-messe au Festival mondial des arts nègres de Dakar (1966), un jeune Dogon préparait un coup d'Etat littéraire. Le silence consterné qui reçut, en Afrique, Le Devoir de violence de Yambo Ouologuem, paru en 1968, fut à la hauteur du retentissement qui accueillit, en France, le premier prix Renaudot décerné à un auteur de l'Afrique subsaharienne. Entreprise de sape de l'idyllisme de l'Afrique ancestrale et de l'illusion décolonisatrice des nouveaux roitelets en costume cravate, la position de brise-fer de tabous du Devoir de violence suscita une levée de sagaies qui lui fut fatale. Après le renversement de Modibo Keita par Moussa Traoré (1968) et la chute de Ouologuem, les velléités de contestation furent contenues pendant une décennie avant une éruption littéraire de voix contestataires : Les Angoisses d'un monde, Le Prix de l'âme, Sahel! Sanglante sécheresse, Les Ruchers de la capitale, Le Fils de chaos, sont quelques uns des titres qui parlent d'eux-mêmes. Palimpseste des souffrances vécues, la littérature malienne commença à oser fissurer les remparts des fors intérieurs. La destitution du président Moussa Traoré en 1991 et l'avènement d'une démocratie multipartite ont ouvert une ère pleine d'espoirs et d'incertitudes. La nouvelle génération d'écrivains, sur les traces de leurs aînés, poursuit la conquête d'un espace public de la parole et travaillent à la 
représentation textuelle de soi. Soit un corpus d'ensemble d'œuvres réalistes qui manifestent un esprit de conciliation critique, à l'exception notoire du Devoir de violence. Bien que la virulence de certains textes-projectiles soit objectivement catapultée contre le miroir aux alouettes d'une gérontocratie traditionniste accrochée coûte que coûte à ses pouvoirs, le recours à la méthode de l'interpellation, sans réponse clairement déclarée, reste la forme privilégiée: "Qui est plus détestable? Celui qui vend son enfant ou celui qui achète un être auquel rien ne le lie?» (Noctuelles : 45). «Qui pourra jamais, un jour, dire les traumatismes des enfants de père polygame?» (Fils de chaos: 105). «Il faudrait trouver des Maliens qui pensent au Mali. La question est : est-ce qu'il en existe?» (Kouty: 78). Ce questionnement de la violence par le parler-vrai, s'il n'est pas exactement subversif demeure transgressif et dérangeant au regard des habitudes.

5 Dérangeante, la violence l'est aussi pour le discours scientifiques. Elle met mal à l'aise les chercheurs qui aspirent à une reconnaissance des cultures africaines, tandis qu'elles sert trop facilement de caution solidaire à ceux qui déprécient les sociétés africaines à l'aune d'une lecture apologétique des régimes démocratiques libéraux, « oubliant » que les sociétés communautaires africaines n'ont pas le privilège du monopole des violences. Le rapport de juillet 2002 de la Commission européenne pour l'égalité des chances établit qu'en Europe les violences domestiques sont pour les femmes de 15 à 44 ans la principale cause de mortalité et d'invalidité, avant le cancer et les accidents de la route...Il importe donc de rejeter toutes comparaisons hâtives, surtout lorsqu'elles opposent des sociétés historiques à une intention, un idéal, telle la Déclaration des Droits de l'Homme où triomphe l'égalité des individus, alors que «l'individu n'est rien dans une société de solidarité " (Noctuelles). Cette réalité de la négation du sujet individuel, il convient de l'intégrer comme une donnée objective afin de ne pas considérer a priori, à la manière de la violence épistémologique du discours colonial (Mbembe, De la postcolonie), cet écart avec les normes de la DDH comme une erreur, une aberration. Ce d'autant plus que la violence est une notion difficile à cerner. L'OMS dans son rapport mondial de la violence de 2002 la définit ainsi : «menace ou utilisation intentionnelle de la force physique ou du pouvoir contre soi-même, contre autrui ou contre un groupe ou une communauté qui entraîne ou risque fortement d'entraîner un traumatisme, un décès, des dommages psychologiques, un mal-développement ou des privations». Malgré sa volonté d'être précise, cette version reste sujette aux représentations socioculturelles. Sur la notion de pouvoir par exemple ou sur l'idée d'intention : celui qui use de la force de son pouvoir n'a pas nécessairement l'intention, ou la conscience, de causer un préjudice mais au contraire de réparer un tort, de prévenir un danger. La perception de la violence est donc soumise à la légitimité que chacun reconnaît, ou non, au pouvoir coercitif du contrôle social, que celui-ci émane d'un Etat, d'une communauté, de sa famille ou d'un individu en particulier. Enfin, pour appréhender les phénomènes des violences familiales, il est peu aisé de distinguer les agents déclencheurs des violences des facteurs amplificateurs. Dans une même famille, les interactions sont complexes entre les facteurs individuels (traits des personnalités), relationnels (entente ou non entre les membres), socio-économiques (conditions de vie, niveau d'éducation), culturels (degré de religiosité, d'attachement aux traditions) mais aussi environnementaux (tension politique, crise économique) et accidentels (ivresse, perte d'un emploi).

Les violences conjugales

6 En vertu du droit coutumier, l'union matrimoniale confère au mari un statut social supérieur à celui de sa femme : les épouses évoquent leur mari, dans les dialogues, en 
terme de «maitre » et de "propriétaire ». En outre, le chef de famille jouit du droit d'infliger des châtiments corporels à sa femme si celle-ci, à son goût, faillit à ses devoirs, à commencer par celui d'obéir. En comparant les différentes scènes de violences conjugales qui jalonnent le quotidien des familles dans les œuvres littéraires, il ressort qu'au cœur des troubles familiaux surgit la question de l'autorité, confondue avec la force et le droit à user de la force. « Mon père, dès qu'il franchissait l'entrée du vestibule [de notre maison], il devenait roi » (Fils du chaos: 15). Les abus d'autorité, dont ce « roi » n'est pas avare envers les gens de sa cour d'habitation, se manifestent par des actes d'intimidation, d'humiliation ou d'agression physique et détériore une situation familiale qui devient celle décrite par Mandé-Alpha Diarra : «la rancœur et la peur rongent l'amour qui unit les miens » (La Nièce de l'imam: 13). S'il est vrai que l'autorité désigne un attribut caractéristique de celui qui commande et impose l'obéissance, ce sens dominant tend à éluder l'autre facette de l'autorité : son sens moral. L'autorité autoritaire qui n'accomplit pas la contre-mesure de son pouvoir (l'autorité morale) dérive vers l'autoritarisme, la tyrannie. Tandis que le détenteur de l'autorité morale aspire à la sagesse qui, traditionnellement, doit guider une communauté vers ce qui est autorisé et bon pour elle, le mari tyrannique n'est lui capable que de réprimer ce qui est interdit. L'un jouit du respect sans la menace et propose un sens à suivre auquel il est lui-même soumis, l'autre n'a que la crainte comme argument et l'arbitraire comme moyen. Du mari au maître, de l'autorité à l'autoritarisme, les motivations de ce glissement vers la violence s'expriment chez les personnages fictionnels de deux manières principales : la colère et le mépris de la femme.

7 Quelles sources alimentent ces colères omniprésentes dans les textes? Les facteurs accidentels tels que l'ivresse ne sont pas les plus fréquents. L'agressivité du milieu environnemental (insécurité alimentaire, violence policière, mépris subi au travail) pèse davantage. "C'est la misère qui rend les gens méchants " (Noctuelles; Sahel!), la misère qui dessinerait le caractère d'époux aussi frustres que frustrés. La colère trahirait l'impuissance sociale extérieure du mari, culturellement tout-puissant à l'intérieur de sa famille. L'extrême pauvreté et la frustration sociale des hommes expliquent-elles cependant que «dans [cette] société, les faibles ne sont pas protégés mais asservis» (Noctuelles: 45) ? De plus, les violences décrites ne touchent pas que les familles pauvres. Des hommes riches et puissants, respectés au sein de leur village, auraient même tendance à recourir à la violence avec d'autant plus de facilité qu'ils se savent craints.

Autant que la misère, l'infériorisation sociale et culturelle de la femme joue un rôle prépondérant dans la mesure où la dynamique des violences augmente ou diminue selon que les "dominées " se soumettent ou résistent à l'autorité maritale. Or, les réactions diffèrent selon les épouses et, chez ces mêmes personnes, varient selon les contextes. Suivant les faits narratifs, la position des femmes est ambiguë : le rapport de force qui les soumet est tout à la fois admis (approbation normative), reproduit (préceptes éducatifs inculqués par les mères aux enfants) et déploré en secret. Il est parfois contesté ouvertement au prix de persécutions. Affronter le chef de famille est perçu comme un affront à l'honneur du nom et du sang de la famille qui se paie cher. La plupart des femmes, enserrées dans un étau, entre d'une part le poids de l'éducation et l'assimilation de normes socioculturelles et d'autre part les risques certains d'humiliation et de châtiments, préfèrent agir comme « Rokya [qui] restait dans les 
normes de son éducation. Son mari était le maître et elle lui devait le respect, même s'il la battait » (Les Ruchers de la capitale : 43).

9 Respect ou crainte? Quoi qu'il en soit, dans toutes les situations où la femme tente de préserver le bon ordre de la famille en s'opposant à son mari, elle est irrémédiablement battue, insultée et le plus souvent répudiée. Elle devient la figure du déshonneur : « des femelles qui ne semblaient pas savoir qu'elles étaient nées pour procréer et obéir » (Prix de l'âme) et qu'il s'agit d'humilier en conséquence. "Pauvres femmes que nous sommes...traitées comme des esclaves, de vils objets sans âme " (L'Héritage empoisonné : 177). La vieille villageoise résignée qui s'exprime ainsi ne comprend pas pour autant l'entêtement de la veuve Bintou qui refuse d'épouser le frère de son mari défunt ainsi que le prévoit la tradition du village. Pour elle, l'attitude de Bintou est celle du déshonneur et de la malédiction. Car la soumission sociale est souvent comprise comme une soumission à Dieu : qui transgresse la norme sociale s'attire les foudres divines. Cela apparaît plus précisément dans les discours tenus aux enfants et dans les explications des accidents. Aussi, rongées par la peur et le doute, beaucoup de femmes s'en tiennent à une observance rigoriste de ce qu'elles croient être la tradition qui les conduira au paradis après leur mort. D'où cette provocation que formule Moussa Konaté : "Vaut-il seulement la peine de compatir à sa misère quand [la femme] elle-même, souvent, semble l'assumer ?» (Fils du chaos : 128).

L'absence des femmes écrivains dans la littérature malienne fait ici particulièrement défaut pour décrire les mécanismes psychologiques et les logiques des femmes dans leur travail de reproduction du modèle social en vigueur. La peur du déshonneur, la peur d'être répudiée et la dépendance économique ne sont-ils que des facteurs amplificateurs? On regrette que Moussa Konaté n'aille pas plus loin - mais le pouvait-il ? - dans Un appel de nuit :

Alima : [papa] a tellement maltraité maman

Doulaye : Est-ce que tu ne t'es jamais demandé pourquoi maman ne se révoltait pas? Alima : Parce qu'elle était faible et analphabète

14 Doulaye: tu sais j'ai rencontré aussi des femmes fortes et instruites qui ne se révoltaient jamais contre leur mari. Ce n'est pas aussi simple. (p.18)

Le verso de la question réside dans ce que prévoient les traditions quand le chef de famille n'assume pas correctement son devoir, qu'il met lui-même en péril sa famille par un comportement colérique et que sa femme refuse d'en subir les conséquences? Aucune des fictions romanesques n'apporte de réponse. Ou plus exactement la littérature tend à montrer que les traditions n'ont pas intégré cette donnée. Ainsi, le narrateur du Fils $d u$ chaos raconte-t-il comment son père répudia sa mère avant qu'une médiation de notables du village ne l'enjoignît à reprendre sa femme: «Mon père a répondu que son épouse devait se mettre en tête qu'elle n'était rien qu'une femme. Ma mère est tombée à genoux, lui a demandé pardon » (p.84). Plus loin dans le récit, un imam face à une femme qui s'opposa à son mari la condamnera du pire mépris : « Honte à toi de n'avoir pas su qui tu es »(p.140). Pourtant, dans les deux cas, il s'agissait de femmes soumises, acceptant le modèle social et subissant les abus d'autorité quotidiens de leurs maris. Puis survint fatalement un jour où ces deux femmes, excédées, se révoltèrent. Face à l'incompréhension générale de leur attitude, elles-mêmes en éprouvèrent du repentir, de la culpabilité. « On peut ressentir de la culpabilité et n'être coupable de rien » souligne le psychiatre Fethi Benslama (2005: 59) 5 . Sauf, objecteront 
les tenants de la tradition, que ces femmes ont outrepassé leurs droits en se rebellant contre leur "maître ». Ces situations révèlent donc une faille dans le système social, une impasse de la réflexion sur ce que sont l'autorité et la tradition (ce mot attrape-tout qui dispense de toute justification), traduits par un refus du dialogue et du débat, ce dont s'agace Ousmane Diarra dans Vieux Lézard: "nos bonnes vieilles traditions bruyamment revendiquées par ceux-là mêmes qui ne se donnent pas la peine de les comprendre (ils ont d'autres chats à fouetter)... Pourtant c'est penser qui transforme les grains de mil en bière, ce n'est pas de moi cet adage bien de chez nous ».

Pourquoi cette esquive, ce refoulement de la question de la fiabilité, la tradition étant invariablement présentée comme infaillible? Que vaut le droit coutumier sans l'application des devoirs et de l'exigence morale sinon une tradition escamotée, un système social censé reposer sur la loi de l'effort commun dérivant vers la loi commune du plus fort? Or, le chef de famille est lui-même reconnu d'emblée comme infaillible : c'est la femme qui a tort, et s'il s'avère que l'homme a commis une erreur, ce sont les agissements mal intentionnés de la femme qui l'auront poussé à la faute. A revers, qu'un homme reconnaisse ses doutes, ou ses erreurs, devant la famille et le voici moqué...par les femmes de sa propre cour d'habitation qui dénoncent un mari "incapable de porter le pantalon", qui s'inquiètent d'un homme "qui porte le pagne "... Ces scènes se répèteraient-elles dans des textes écrits par des femmes ou révèlent-elles en filigrane les peurs des auteurs masculins?

Le pouvoir des femmes sur leurs maris est en effet une nouvelle donne sociale qui émerge dans la littérature malienne de ces dernières années. Ce changement dans le rapport de force du couple reste néanmoins confiné au cadre des familles aisées de Bamako. A la différence des femmes villageoises, les femmes de la ville peuvent échapper plus facilement au contrôle social, espérer de nouvelles rencontres. Souvre ainsi, la possibilité de changer de vie en changeant de mari. La ville devient le lieu des « cocufiés cocufiant » selon l'expression de Moussa Konaté (Prix de l'âme) et les charmes féminins, une arme pour faire pression sur les maris qui négligeraient, financièrement, leurs femmes. L'espoir de la réussite sociale et l'appât du gain modifient les perspectives et les comportements de femmes qui ne se voient plus pour seul horizon possible, l'unique dignité de la maternité.

Plus généralement, la lutte des femmes pour acquérir et préserver une place sociale honorable se révèle au fil des textes particulièrement âpre. Mêmes malheureuses avec leur mari, elles se battent pour ne pas être répudiées ou isolées. Avec la polygamie, le combat se situe au cœur même de la famille : rivalités et coups tordus entre coépouses enveniment la vie quotidienne. Ainsi une mère confie-t-elle à son fils : « quand ton père t'a battu et chassé, ils ont ri Farima [la coépouse] et ses enfants. Ils n'attendaient que ça. Farima a usé de tous les moyens pour me rendre antipathique à ton père " (Fils $d u$ chaos : 62). La coépouse censée être la "petite sœur » devient une rivale. L'une l'autre souffrent des privilèges ou se réjouissent des malheurs de l'autre : «l'altercation entre Hawa et sa fille s'entendait bien dans la cour. La coépouse Amina était folle de joie : Mariama n'était pas heureuse » (Toiles d'araignées : 56).

Par ailleurs, les représentations littéraires mettent en avant l'importance du soupçon d'intentionnalité : « je vis déjà avec la hantise de voir mon mari choisir une coépouse, rien que pour m'embêter et me rendre la vie impossible» (Un héritage empoisonné: 16). Qu'une femme n'apprécie pas que son mari convole avec une autre femme n'a rien de surprenant. En revanche, qu'elle se persuade qu'il le fasse uniquement pour nuire à son 
bonheur, voilà qui est plus singulier. Des études psychiques sur les processus sociocognitifs ${ }^{6}$ montrent que dans une situation d'agression, ou perçue comme telle, si un individu attribue le comportement agressif à des facteurs accidentels, il réagira de manière moins agressive que si le comportement est attribué à une intention volontaire. Le soupçon d'intentionnalité favorise une interprétation hostile, voire paranoïaque, des faits et paroles de chacun, sur laquelle se greffent aisément les "superstitions» (terme employé par les écrivains). Aussi, les échanges entre deux coépouses rivales ressemblent souvent à une rude compétition de sortilèges et d'antidotes, via l'entremise de féticheurs en tout genre. Dès lors, dans « une vie familiale faite de rancune et de jalousie " où «tout le monde se méfiait de tout le monde " (Fils du chaos: 73, 144), le déclenchement des violences n'attend qu'un geste qui pourrait agréer la suspicion. Au mari « fou de colère » succèdent dans les violences familiales les épouses «folles de rage » qui, faute de pouvoir se retourner contre leur mari, déchargent leur agressivité à l'encontre des coépouses, des enfants ou des travailleurs domestiques.

20 A travers le thème des violences conjugales, les œuvres contemporaines reprennent, dans un tout autre langage, un des principaux motifs des littératures orales: dans une culture fondée sur une communauté sociale inégalitaire, si l'autorité n'est pas caractérisée par la pondération et la concertation, germe alors en lieu et place un ersatz dangereux de la tradition, en proie aux caprices et faiblesses de ces détenteurs.

La femme maudite

21 " Au pays des hyènes, on a intérêt à ne pas bêler, même si on est agneau » (Vieux Lézard). La peur qui couve et commande les échanges familiaux génère plus de frustrations que d'explications, plus de suspicion que de compréhension entre les individus. D'où la nécessité de boucs émissaires comme exutoire collectif aux refoulements individuels. Ceux-ci ne s'incarnent pas seulement chez les « déviants » du système mais aussi à travers différentes figures accidentelles. Les femmes, "piétinées par la vie» (Toiles d'araignées: 12), ne sont pas seulement victimes des violences conjugales, mais les plus exposées à l'exclusion, accusées d'être responsables du malheur familial : «Mon père m'a toujours dit : méfie-toi de la femme car c'est par elle que Satan entre dans les familles » (Un Appel de nuit:12). M.B. Diallo souligne que si la femme dans la littérature africaine est « tour à tour idéalisée et célébrée ; elle n'en est

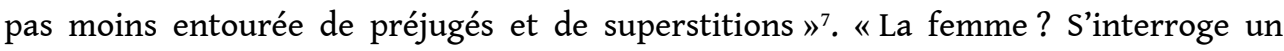
garde-cercle : Quelle obscurité ! (Le Lieutenant de Kouta : 34) Loin d'être un phénomène folklorique secondaire, le gouvernement invisible des puissances surnaturelles ne règne pas seulement dans quelques esprits impressionnables, mais préoccupe une large partie de la population "qui croit que des êtres invisibles et malfaisants peuplent l'univers" (Fils du chaos: 169) et régit de manière péremptoire le traitement des malheurs de la vie. Dans Les Angoisses d'un monde, "partout où l'activité quotidienne pouvait s'exercer, les gris-gris, amulettes et talismans étaient des alliés sûrs,..., rien ne permettant de savoir d'où viendrait le danger, l'on passait son temps à surveiller l'attitude de ses voisins et à interpréter leurs paroles» (p.10). A la recherche des causes factuelles se substitue une logique « extérieure » qui se manifeste en premier lieu par la malédiction dont est censée être frappée la personne touchée par un incident antisocial. Ainsi, tous les dialogues littéraires sont-ils envahis par les termes "maudit(e)s » et «malédiction » : une femme stérile : maudite! Une jeune fille violée : maudite! Une jeune veuve : malédiction! 

efficace des vœux» (Les Angoisses: 30). «Parle jeune homme! S'exclame un grand marabout de la capitale. Ta bouche a tété le sein d'une femme soumise, donc bénie » (Nièce de l'imam: 85). Maudite donc la femme qui se rebelle. Dans Un Appel de nuit, lorsque les parents émigrés en France décident de retourner au Mali, Alima refuse de les suivre. Devant la détermination de sa fille, la mère s'exclame : «Une fille maudite, voilà ce que tu es ». Le refuge dans la malédiction se révèle être une fuite en avant pour ne pas chercher les raisons véritables d'un comportement. Evitement de la remise en cause, recherche d'attribution à des phénomènes extérieurs caractérisent également cette scène de Toiles d'araignées lorsqu'une mère tente d'abord une médiation auprès de Mariama pour la convaincre d'épouser le vieux Diallo, puis se persuade qu'il s'agit d'un mauvais sort jetée sur sa fille par la coépouse. Dès lors, au lieu de poursuivre le dialogue, elle s'en remet à un "charlatan" (sic) pour désenvoûter Mariama. Ce scénario-type réapparaît souvent dans les textes. M.-A. Diarra évoque «la loterie divine » comme sport national tandis que $\mathrm{M}$. Konaté ironise : « seuls les sortilèges et les marabouts étaient capables de conjurer le destin, de faire réfléchir Allah qui, souvent, à cause de l'immensité et de la complexité de sa tâche, se trompe " (Fils du chaos: 86). Outre, que les procédés de l'intercession maraboutique aboutissent rarement, cette voie ouvre une impasse pour la raison critique qui ruine la concorde des familles les plus paisibles. Il n'est plus question de discuter, de convaincre, ni même de comprendre, il faut extirper le mal par l'action, extraire de la famille ce danger, soit concrètement: rejeter, exclure, bannir. La malédiction se présente aussi sous les aspects de celles et ceux réputés être réprouvés de Dieu. Ainsi, la stérilité d'une femme déclenche moins la compassion que la médisance et le rejet. Quand Founéba, première épouse du chef du village, fut déclarée stérile « tout Willimano chuchotait alors qu'elle portait malheur » (Prix de l'âme: 19). Déconsidérée dans la famille, moquée dans le village, celle-ci se sentit de surcroît coupable et punie par la colère divine. Cette assimilation de la maternité à la respectabilité sociale est représentée d'une manière extrême dans $L a$ Nièce de l'imam: "Un midi, [son nouveau mari] la viola. Fatima en fut horriblement malade mais en conçut une grossesse. De joie, elle en oublia l'horreur du viol» (p.50). L'auteur est-il crédible? Si oui, c'est une violente démonstration de la déconsidération de soi au profit du statut social, de la maternité comme soulagement d'échapper à l'enfer des femmes stériles.

La peur du déshonneur apparaît du reste dans l'ensemble des œuvres littéraires comme un fléau social. Non seulement parce qu'elle conduit à l'exclusion mais parce qu'elle offre à la rumeur une puissance de contrôle social incontrôlable et à la médisance un vrai pouvoir de nuisance. Après que Bata dut se résoudre à quitter son villace, une femme lui confie: "On est allé dire beaucoup de mal de toi, Bata. Ta mère en est tombée malade. Elle t'a maudit " (Une Aube incertaine: 154). Lorsque "radio-trottoir " (M.A. Diarra) ou « radio-duruni $»^{8}$ (I.S.Traoré) montre du doigt une famille, la pression sociale qui s'exerce alors sur le chef de famille le conduit à réagir de façon radicale contre le membre de la famille sur qui la rumeur s'est abattue. Cette situation est particulièrement probante dans le cas des filles-mères victimes de viols. La jeune fille n'est pas une victime qu'il s'agirait de protéger mais une "maudite ", une "bâtarde " (autre expression répandue) qui afflige, humilie et déshonore la famille et son ascendance. Les souffrances physiques et psychologiques endurées par la jeune fille ne comptent pas à côté de la violation de la tradition. Peu évoqué dans la littérature, ce sujet a néanmoins fait l'objet d'un film, Den Muso (La jeune fille) de Souleymane Cissé 
qui déclarait: "J'ai voulu exposer le cas de nombreuses jeunes filles rejetées de partout. J'ai voulu mon héroïne muette pour symboliser une évidence : chez nous les femmes n'ont pas la parole $»^{9}$. La littérature ne raconte pas non plus, en dehors d'une brève évocation dans Le Devoir de violence de Yambo Ouologuem, le cas particulier des jeunes filles « offertes » aux administrateurs coloniaux en tournée dans les villages. Qui étaient-elles pour qu'on les sacrifie? Que devenaient-elles après le passage du « commandant»?... Des maudites?

Pour comprendre les superstitions qui entourent les femmes stériles ou les femmes violées, la notion d'accident associée à la volonté divine de punir des pécheresses intervient comme une explication admise au sein des familles. Les femmes qui perdent un enfant jeune subissent les mêmes suspicions. La malédiction se substitue au malheur et révèle l'impensé des déviances accidentelles. Lorsqu'une jeune fille doit épouser un septuagénaire maladif (mais riche), il est parfaitement «naturel » que le mari meure dans un temps plus ou moins court après le mariage. Ici, la notion d'accident ne devrait donc pas exister, sauf à soupçonner la jeune épouse de meurtre. Pourtant, les jeunes veuves, en particulier celles qui n'ont pas eu «l'honneur » de la grossesse, deviennent à leur tour des femmes maudites, «des enterreuses" (L'Héritage empoisonné). Dans La Nièce de l'imam, Fatima crut d'abord échapper au sort des jeunes veuves grâce au désir de Bilaÿ de la prendre pour épouse. Malgré l'incompréhension des villageois, elle lui fut donnée en mariage. Hélas pour la jeune femme, elle fut victime d'un accident alors qu'elle était enceinte et perdit son enfant. Aussitôt, le marabout du village expliqua à Bilaÿ que sa femme était «marquée du sceau fatal des veuves exterminatrices» (p.51). Fatima fut donc abandonnée.

A dire vrai, les œuvres littéraires ne fournissent pas d'indices probants pour comprendre ces croyances, mais elles éclairent la déresponsabilisation de la communauté. La soumission de la raison de causalité et des logiques historiques aux mystères surnaturels, c'est d'abord l'abandon de la responsabilité. Et la femme violée est alors plus coupable le violeur! Se confronter à l'obligation de penser et prévoir l'intégration des phénomènes humains accidentels, comme celui des femmes violées, exigerait par ailleurs une véritable révolution copernicienne de la vision de la femme. Pour l'heure, les femmes, villageoises en particuliers, « restent toujours mineures aux yeux de leur entourage (...) toujours soumise à la volonté de ceux qui les maintiennent dans cette situation d'éternelle enfant" (Un Héritage empoisonné: 26). Ce n'est pas l'imam de Fils du chaos qui le contredira considérant que « la femme est ce qu'elle est: quelque soit son âge, elle demeurera toujours un enfant » (p.138).

L'enfant au front des batailles familiales

La famille, cellule de base de la socialisation, constitue le lieu central de l'intimité et du développement des enfants. Lorsque le milieu familial souffre de l'intrusion de la violence en son sein, l'enfant en est le premier récepteur : soit qu'il subit lui-même des violences physiques, verbales ou psychologiques, soit qu'il assiste à celles commises à l'encontre de sa mère et/ou des autres femmes présentes dans le lieu d'habitation familial. «L'amour d'un enfant pour ses parents n'a besoin de nulle manifestation (...). L'obéissance seule est permise, souhaitée, exigée " (Toiles d'araignées: 59). Cette conception éducative est retranscrite dans les dialogues par une violence verbale terrifiante : «Si tu n'agis pas selon sa volonté, [ton père] te tuera, et moi [ta mère] je n'y pourrais rien. (...) il a droit de vie et de mort sur nous tous » (Fils du chaos: 61). Un père rappelle sa fille à l'ordre : «Alima prends garde : le nom, l'honneur, le sang de 
notre famille sont plus précieux que ta vie " (Un Appel de nuit : 13). Ou bien une femme trop pauvre pour élever son enfant donne son fils à un notable : «Considère-le comme le tien. Bats-le s'il te désobéit, tues-le s'il ne mérite de vivre: je ne t'en demanderai même pas compte " (Une Aube incertaine : 27).

Dans les œuvres de Moussa Konaté, on est loin du bienheureux Enfant noir de Camara Laye ! « [Enfant], la terreur me cernait. Et personne ne soupçonnait mon trouble » (Fils $d u$ chaos : 64). De plus, l'enfant tenté de désobéir entend se dresser les mêmes démons culpabilisants de la malédiction, en sus des coups de poing ou de trique reçus sans ménagement, quand il ne devient pas le souffre-douleur de la famille, le bouc émissaire du père colérique ou de la mère qui reporte sur lui ses propres violences subies. Ainsi Antoinette qui «ne pouvant assouvir sa haine en molestant [les hommes qui l'ont violentée], se rabattit sur son enfant qui, dès lors, ne connut plus un instant de paix " (Une Aube incertaine : 88).

Par ailleurs, M. Konaté et I. Ly montrent que les enfants subissent les violences ordinaires que provoquent les rivalités et jalousies entre les épouses d'hommes polygames. Toiles d'araignées décrit des scènes de joie et de tendresse entre Mariama, petite fille, et son père. Puis « la vie changea quand une seconde épouse entra dans la maison (...). Mariama subit toutes les avanies de sa marâtre sans que son père osât bouger » (pp. 49-50). Ailleurs, «[la] mère et sa coépouse s'insultaient volontiers, en venaient aux mains. Naturellement, les enfants aussi entraient dans la danse » (Fils $d u$ chaos: 73). Notons que l'enfance des fillettes est quasiment absente des textes (en dehors de sa fin brutale avec les mariages forcés précoces). Est-ce parce que la quasi totalité des écrivains maliens sont des hommes? Toujours est-il que ni les mutilations sexuelles (sauf allusion dans La Nièce de l'imam), ni l'inceste ne font l'objet de récit. De même, le placement des enfants chez un parent lointain n'est pas évoqué. Enfin, seul I.S. Traoré, en introduction de son dernier roman Les Amants de l'Esclaverie, évoque la mise en gage d'enfants par les parents, appelé le tonomada au Mali, qui consiste à envoyer un enfant travailler dans une autre famille auprès de laquelle les parents ont contracté une dette.

Les traumatismes infantiles apparaissent donc mésestimés. En revanche, ils hantent les œuvres de Moussa Konaté : «Se peut-il que tu n'oublies jamais ton enfance? / Je ne sais pas. Ça me revient toujours à l'esprit. Je ne sais pas pourquoi ?» (Une Aube incertaine : 165). Dans Le Prix de l'âme, Djigui grandit avec «l'image indélébile du viol de sa mère [par son père] » (p. 24). Quant au Fils du chaos, il ne cesse de se demander : "Qui pourra jamais évaluer les conséquences de ces traumatismes sur la société des adultes?» (p. 105). Intériorisation de l'agressivité, révolte inhibée, violence enkystée dans les profondeurs de la conscience : " [Nos jeunes] sont fils de la femme et de la rancœur » affirme I.S. Traoré (Les Amants de l'Esclaverie : 25). La révolte des jeunes, souvent mis sur le compte du déclin des traditions provoqué par la colonisation hier et la mondialisation aujourd'hui, est une "constante dans les récits fondateurs bambara et malinké » rappelle l'auteur (p. 9), parce que seul moyen de lever le poids des pères lorsque la gérontocratie refuse la passation des pouvoirs. "Qui es-tu? / Je suis le fils de mon père / Mais encore? / Je l'ignore moi-même, j'attends de le savoir. Mon père occupe beaucoup de place» (p. 18). De même dans les œuvres de Massa Makan Diabaté, se pose la question de la relation filiale autour de cet adage : Il est trois sortes de fils : celui qui n'atteint pas la renommée de son père; celui qui l'égale; celui qui le surpasse. Mais ce 
dépassement du père tend à revêtir des formes pathologiques chez des enfants maltraités. «Certains jours,... j’ai pensé tuer papa » (Un Appel de nuit : 10).

Le meurtre du père obsède les personnages de $M$. Konaté qui ne parviennent pas cependant à s'y résoudre, sauf dans Les Saisons. Or, nul complexe oedipien dans ce désir de tuer: ce n'est pas l'affirmation du sujet sexué qui s'exprime mais une violence justicière, celle qui pousse la victime d'une injustice non reconnue à obtenir elle-même réparation, comme Kouty, l'adolescente meurtrière du roman d'Aïda Mady Diallo. De manière moins spectaculaire, les traumatismes infantiles liés aux violences familiales jouent un rôle insidieux dans la dégradation du système social puisque les enfants devenus adultes auront tendance à répéter les violences vécues ou à reproduire des logiques comportementales persécutrices. Ils expliquent en partie les faits narratifs observés à propos des violences conjugales. "Une femme n'est rien qu'une femme, avait affirmé mon père, et ma mère lui avait donné raison. Cette pénible scène je ne l'ai jamais oubliée. Maintenant encore, chaque fois que je m'efforce de voir en la femme autre chose qu'une femelle, l'image de ma mère à genoux devant son mari me revient en mémoire et m'ôte toute illusion » (p. 84) soupire le narrateur du Fils du chaos qui se remémore son enfance: "Je suis entrée dans la cuisine. Ma mère y était: j'ai fait semblant de ne pas la voir (...). Triomphant, je suis sorti sans lui répondre parce que je savais qu'elle n'était qu'une femme, rien qu'une femme» (p. 28). Enfin, du premier roman malien, La Passion de Djimé de Fily Dabo Sissoko (1956), au prochain à paraître, Vieux Lézard d'Ousmane Diarra (février 2006), le droit à la différence, étape préliminaire à la liberté individuelle, est revendiqué avec constance. De fait, la société malienne présente un paradoxe: haut lieu de la culture orale, elle prive une majorité de ses membres de la parole.

Le droit d'expression est réservé à une poignée d'hommes et de femmes qui parlent au nom de tous. Cette incommunicabilité offre un terreau fertile pour la rancœur, les pensées soupçonneuses, vindicatives, maudissant, qui vampirisent la raison et les discours. L'exemple type de la convergence des violences parentales et sociales sur une jeune fille est sans doute le mariage forcé avec un vieil homme, thème récurrent des œuvres littéraires, depuis Sous l'orage (1957) à nos jours. Mais il n'y a que dans le roman de Seydou Badian que l'issue est heureuse. Dans les autres récits, la jeune fille qui manifeste son refus subit alors une véritable répression dont le cas de Mariama, la malheureuse héroïne de Toiles d'araignées, dépasse l'entendement, lesquelles représailles ne doivent pas occulter le point de départ du cauchemar de Mariama dont le refus de devenir la coépouse d'un septuagénaire malade ne surgit qu'après le désir de son père de la marier à un riche marchand afin de pouvoir se payer une fillette en nouvelles noces. Au passage, l'auteur ne manque pas de souligner l'influence de la mère, malheureuse épouse, qui pousse néanmoins sa fille à suivre la même voie qu'elle. Quand Mariama lui annonce son intention de refuser le mariage, la mère, avant de la maudire, lui lance : «Mesures-tu ce que ton refus signifierait pour moi ?» (p. 67). I. Ly touche un élément de pression sociale singulièrement culpabilisant pour qui se révolte contre l'ordre établi : en outrepassant les normes, il crée du tort à sa propre famille. Les mères ne cessent de le rappeler à leurs enfants: s'ils ne suivent pas la voie des traditions, ils détruiront l'honneur et la réputation de leurs parents. Dans Fils du chaos, un mère implore son fils d'obéir à son père : «Si tu ne fais pas ce que te demande ton père, il croira que c'est moi qui te pousse à lui désobéir. Il n'hésitera pas à me répudier » (p. 61). Ce qui se produit dans Une aube incertaine. 

un dilemme cornélien. "A quoi bon? Pourquoi vivre alors qu'il n'est plus possible d'être soi-même? » (Sous l'orage: 59). Cette interrogation de Kany dans la tourmente souligne qu'il n'y a pas de suicidé(e) en littérature. Soumission ou fuite sont seules envisagées. En revanche, les mariages forcés d'adolescentes avec des septuagénaires sont saisis par les écrivains pour dénoncer des violences sexuelles, qu'ils n'ont pas évoqué ou oser évoquer au sujet des violences conjugales. En effet, la défloraison virginale est le plus souvent montrée comme un passage traumatique. «A soixante-dix ans, enlever à une pucelle son innocence! Certes la lame du rasoir pourra toujours aider et l'honneur restera sauf " (Toiles d'araignées: 60). Lorsque le vieux Boura voulut consommer le mariage avec Fatima, il s'aperçut qu'elle n'était pas excisée. Sur-le-champ, on recourut à l'ablation de l'organe, après quoi Boura revint dans la chambre de Fatima. Suite à cet épisode, la jeune femme «ne pouvait imaginer un sexe d'homme qu'associé à la lame de l'excision, à l'hernie et aux serres de Boura » (Nièce de l'imam: 60). Quant au vieux Bakary, s'il n'eut pas besoin de la lame pour prouver sa puissance sexuelle et violer Mariama, il bénéficia néanmoins de la complicité de l'oncle de l'adolescente pour l'empêcher de se débattre et s'enfuir. Dans le cas du mariage d'une adolescente avec un grabataire, la violence parentale est totale puisqu'ils condamnent leur enfant au sort des « veuves exterminatrices». Sans se précipiter dans le jugement et la condamnation, l'attitude des parents mérite pour le moins qu'on s'interroge. Parviennent-ils vraiment sans états d'âme à justifier au seul nom de la tradition cet abandon aux malheurs de leur enfant?

La foi en l'autorité traditionnelle est-elle si puissante qu'elle ne suscite chez le père et la mère que le soulagement du devoir accompli et de l'honneur social préservé ? Accomplissent-ils leur devoir, parfois jusqu'au meurtre de leur enfant, avec autant de certitudes que celles décrites? Quid de l'amour filial? Ne sont-ils pas eux-mêmes confrontés à des dilemmes autodestructeurs? Ne « s'exécutent-ils " pas la mort dans l'âme? Les tumeurs - tu meurs ? - de la violence familiale sont sans doute aussi profondes que le refoulement du sentiment amoureux: "Je ne me suis jamais demandée si j'aimais Bilaÿ ou non... Il n'est pas question d'amour, mais de cohésion de la communauté... Ces questions d'amour du couple sont pour ta génération » (Nièce de l'imam). «L'amour serait-il une invention de notre génération?» feint de se demander Moussa Konaté (Fils du chaos).

Les violences familiales dont sont témoins les enfants ou qu'ils endurent comme victimes directes sont dénoncées dans le rapport mondial sur la violence de l'OMS (2002) comme étant responsables des premiers troubles pathologiques d'une société, quelle qu'elle soit. Les communautés africaines peuvent-elles faire l'économie de cette réflexion sur les violences sociales au prétexte qu'elles sont motivées par les bienfaits de la tradition? Qu'est-ce donc qu'une tradition: les fondements historiques d'un modèle social en évolution ou l'imitation sociale irréfléchie d'un modèle historique figé ? La première hypothèse se traduit par un continuum culturel, une prolongation historique dans les adaptations contemporaines (plus couramment appeler développement endogène). La seconde transforme la notion de cohésion sociale en ordre totalitaire, ce que Montesquieu résumait ainsi : «la loi faite pour nous rendre plus juste ne sert souvent qu'à nous rendre plus coupables » (Lettres persanes). Face aux avanies des violences familiales, l'enfant n'a d'autre droit que celui se taire : il s'écrase. Comment grandir, s'épanouir, quand sa propre dimension est réduite au silence?

Bulletin de l'APAD, 27-28 | 2008 
Qui sème le vent...

«Bissimilaï! Ou le monde s'est retourné ou je sors d'un mauvais rêve » (Le Boucher de Kouta: 69). Les textes littéraires décrivent une communauté qui souffre d'une socialisation corrompue à outrance, voire d'une socialisation à outrages lorsque se substitue à la perte des repères objectifs et positifs, la violence comme mode de régulation sociale. Si les situations de violence racontées ne sont pas spécifiques au Mali, ni même à l'Afrique, elles donnent néanmoins au lecteur le sentiment d'une société malienne traumatisée.

Prenons un extrait d'Une aube incertaine de Moussa Konaté, condensé exemplaire des situations décrites au cours de notre étude (violences physiques, mépris de l'homme pour la femme, rivalités entre femmes, autoritarisme, absence de dialogue, sacralisation des hiérarchies, absence de contre-pouvoir, peur, incompréhension, défense et reproduction du modèle sociale par les victimes, malédiction, rancœur vindicative, exclusion, dilemme psychologique, etc.) : Bata s'était réveillée bien avant l'aube, ... elle travailla tant qu'à midi, elle éprouvait de la peine à se tenir debout...Bata dut peiner ainsi jusque tard dans la nuit...puis elle s'endormit aussitôt sur son tara. Pour la première fois depuis qu'elle [travaillait] chez Faran, elle se réveilla après le soleil. Les maîtresses de maison ne se gênèrent pas pour lui dire ce qu'elles pensaient. Insultée, moquée, étourdie, Bata oublia son premier devoir quotidien : aller devant la porte de la chambre de Faran et, un genou à terre, dire : Bonjour, mon maître. Faran fit appeler Bata...Alors, tu ne me salues plus ? ...Faran lui ordonna de se mettre à genoux : il la battit avec son nerf de bœuf...Plus tard, Waly [mari de Bata] alla trouver son père adoptif dans la hutte où il trônait. Pourquoi as-tu frappé Bata? Faran regardait Waly avec dédain. Pourquoi l'as-tu frappée? Est-ce que tu es son mari ? Dans la famille régnait un silence de mort, tant la stupeur était grande : jamais personne n'avait osé s'adresser au chef avec tant d'insolence.

\section{Va-t'en d'ici, chien! Vociféra Faran}

Je te demande pourquoi tu l'as battue. C'est ma femme ! Cria Waly

C'est moi qui te l'ai donnée, maudit ! Sans moi, que serais-tu?

[Waly gifla le vieillard. Il fut alors ligoté et battu à son tour]. Ecartant la foule, la mère de Waly s'approcha de son enfant couché à terre. Elle le regarda longuement et dit : Ah! Waly, tu m'as tuée! Elle franchit de nouveau la foule, disparut... Bata et Waly comprirent, sans se le dire, qu'ils n'étaient que des serfs aux mains de Faran. Je le tuerai, je le tuerai, murmurait Waly entre ses lèvres tuméfiées. A l'aube, les deux jeunes gens prirent le chemin [de l'exil]. (pp. 28-30)

4 [Quelques années plus tard, une femme du village croise Bata en ville]. C'est toi Bata? Ah! Quel malheur t'es arrivé ? Tu es partie comme ça sans le consentement de ta mère...Elle a beaucoup souffert ta mère. Pourquoi as-tu suivi cet ingrat de Waly qui a osé gifler Faran son bienfaiteur ?... Tu sais quand tu es partie ton père a répudié ta mère...quand une femme a subi cette épreuve sous le regard de ses coépouses, elle ne peut plus relever la tête. Ton père avait même juré qu'il te tuerait... Je me demande chaque fois que je pense à toi : Que lui est-il arrivé ?... On est allé dire beaucoup de mal de toi, Bata. Ta mère en est tombée malade. Elle t'a maudit, elle qui t'aimait tant, et elle ne veut même plus entendre parler de toi. (pp.153-154)

Qui sème le vent récolte la tempête, telle pourrait être la morale de ce récit qui dit assez la complexité des divers points de vue et des incompréhensions qui n'attribuent 
pas aux mêmes causes la responsabilité de cette histoire dramatique. En effet, qui sème ici le trouble? Toute réponse ne peut que trahir une perception socioculturelle subjective. Doulaye Konaté, qui plaide en faveur des mécanismes traditionnels de prévention des conflits, rappelle l'importance de la notion de consensus dans la culture malienne. "On pourrait objecter, écrit-il, que le consensus n'est pas synonyme de démocratie. Soit, mais faudrait-il pour autant occulter les formes de concertation au profit des «caricatures » de procédures dites démocratiques? $»^{10}$ La question est en effet d'actualité. Mais cette remarque confond actes électoraux et espace public, droit de vote et droit d'expression. De plus, le consensus s'établit souvent au détriment de la justice, la cohésion du groupe primant sur les réparations individuelles. Or, les récits littéraires mettent justement en lumière comment la famille et la communauté sont mises en danger par les répercussions néfastes des sentiments d'injustices individuels et comment l'évitement des situations conflictuelles ouvertes sourd de la violence.

Comment sortir des dynamiques des violences familiales? Par un placage des Droits de l'Homme comme solution finale aux cultures communautaires? Les écrivains pour leur part font ressortir trois priorités. Repenser l'autorité, ses moyens et ses objectifs. Réajuster la place de l'individu dans la communauté en respectant son intégrité physique et morale. Vaincre l'idée de la «malédiction » et reprendre confiance en soi. Bien sûr, chaque écrivain aurait d'autres vœux à ajouter. Moussa Konaté demanderait l'abrogation de la polygamie, Ibrahima Ly et Ismaïla Samba Traoré insisteraient sur la nécessité de rétablir la vérité historique des siècles écoulés, Ousmane Diarra et Aïda Mady Diallo revendiqueraient le droit à l'individualisme. En attendant, tous assurément réclament le droit à la liberté de parole et au débat pour que crèvent les plaies qui gangrènent le dynamisme et la confiance des Maliens. La déresponsabilisation et la déconsidération de soi engendrent des conséquences sur les individus et la société encore trop mésestimées. L'importance, parfois outrancière, accordée aux richesses du passé, à l'honneur et à la réputation traduit aussi la recherche à tout prix d'une dignité à retrouver. A défaut, l'humour vient souvent comme une béquille : On dit que lorsque Dé Gôli vit son pays envahi par les Allemands, il vint voir Allah... Dé Gôli gagna la guerre. Quand Krouchef était dans la pagaille semée par Staline, il est allé voir Allah pour l'inspirer... Et Kennedy aussi était venu, Senghor et beaucoup d'autres. Allah leur a dit à tous que leurs pays auraient beaucoup de richesses...Quand le Maréchal est venu pour savoir seulement si les habitants du Vieux Pays [Mali] pourraient un jour manger à leur faim, avant même qu'il ne dise quelque chose, Dieu secoua la tête et pleura. (Ruchers de la capitale)

Derrière le rire se cache la peur. Jusqu'à la peur d'être abandonné des dieux. La peur d'être un peuple maudit. La peur de soi aussi. «La grande majorité des Africains n'ont pas de réponses aux questions suivantes : où en sommes-nous ? Que nous est-il arrivé ? Que se passe-t-il en ce moment précis de l'histoire de notre continent et de l'humanité ? Comment nous faut-il envisager l'avenir? Telle une chape de plomb, la langue de bois pèse sur les causes des événements passés et présents. ${ }^{11}$ Où l'on retrouve l'absence de débats publics au Mali. Car il n'est pas que le discours littéraire qui contrevienne aux habitudes de la parole voilée, du non-dit et du musalaka. La parole scientifique aussi dérange le "clergé » social et intellectuel établi. Les écrivains et chercheurs africains qui exigent une relecture de l'héritage du passé et un état des lieux présents sont discrédités, privés d'accès à la parole publique. La profession d'un devoir d'inventaire est vécue ou prétendument vécue comme la profanation des mémoires et des coutumes. Entre la défense des traditions et les interdits coutumiers : les fruits défendus 
des cultures traditionnelles ne servent-ils pas de paravent pour dissimuler la tentation de se défiler au lieu de dénouer les fils de l'histoire? Les écrivains contestataires, eux, sont perçus comme Waly et Bata : ils font honte. Entre eux et la société malienne s'est établie une relation fêlée. Or, si l'écriture est une découverte de son «moi " propre, voire une naissance de soi, la littérature est sollicitude. En l'absence d'un public, elle sanctionne ses créateurs d'une longue peine de solitude.

Le Mali vit-il socialement en paix ou est-ce une poudrière qui attend l'étincelle? L'apparente paix sociale du pays se fonde-t-elle sur le sacrifice d'une grande partie de sa population? Ce modèle perdurera-t-il avec l'amélioration économique attendue et les progrès de l'éducation nationale ? Les femmes accepteront-elles encore longtemps d'être infériorisées? L'évolution de la société malienne est-elle une expérience in vivo d'adaptation à la mondialisation forcée d'une communauté attachée à ses valeurs historiques ou un enfermement corrupteur, prétendument traditionaliste, au profit d'une minorité ? L'évolution de cette paix sociale passera-t-elle par un processus endogène progressif, par l'imitation d'un modèle extérieur imposé ou par une révolution? Pour espérer avoir le luxe de choisir au lieu de subir, ne vaudrait-il pas la peine que les Maliens entre eux puissent en discuter ouvertement? A moins de se persuader que ce serait là l'étincelle qui mettrait le feu aux poudres, si les débats tournaient à la discorde ou au vent de paroles. Qui sème le vent...

\section{BIBLIOGRAPHIE}

Badian, Seydou, 1963, Sous l'orage, Présence Africaine, [1957].

Couloubaly, Pascal Baba, 1998, Les Angoisses d'un monde, Bamako, Jamana, [1980].

Diabate, Massa Makan, 2002, Le Lieutenant de Kouta, Paris, Hatier, [1979].

Diabate, Massa Makan, 2002, Le Coiffeur de Kouta, Paris, Hatier, [1980].

Diabate, Massa Makan, 2002, Le Boucher de Kouta, Paris, Hatier, [1982].

Diallo, Aïda Mady, 2002, Kouty, mémoire de sang, Série-noire Paris, Gallimard.

Diallo, Aïda Mady, 2002, Dandara, in Nouvelles voix d'Afrique, Paris, Hoëbeke.

Diallo, Aly, 2000,La révolte du Kòmò, Continents- noirs, Paris, Gallimard.

Diallo, Mamadou Bani, 1998, La femme dans la littérature africaine, in Tapama, n 3, déc. 1998, éd.

Donniya, Bamako.

Diarra, Mandé- Alpha, 1981, Sahel! Sanglante sécheresse, Paris, Présence Africaine.

Diarra, Mandé- Alpha, 1994, La Nièce de l'imam, Saint- Maur- Bamako, Sépia- Jamana.

Diarra, Ousmane, 2002, La côte d'Adam, in Nouvelles voix d'Afrique, Paris, Hoëbeke.

Diarra, Ousmane, 2006, Vieux lézard, Paris, Gallimard.

Fisher, Gustave-Nicolas, 2003, Psychologie des violences sociales, Paris, Dunod. 
Garnier, Xavier, 2002, in Notre Librairie, n¹48, Penser la violence, juillet-sept 2002.

Jeune Afrique-L'Intelligent, n²327, 14 août 2005.

Konare Alpha Oumar, 1997, in Bouillon de culture, « spécial Mali », France 2, enregistré en public le $1^{\text {er }}$ décembre 1997

Konate, Doulaye, 1999, Les fondements endogènes d'une culture de paix au Mali,

www.unesco.org/ccp/publications

Traore, Aminata Draman, 2002, Le viol de l'imaginaire, Paris, Actes Sud-Fayard.

Konate, Moussa, 1981, Le Prix de l'âme, Paris, Présence Africaine.

Konate, Moussa, 1985, Une Aube incertaine, Présence Africaine, Paris.

Konate, Moussa, 1986, Fils du chaos, Paris, L'Harmattan.

Konate, Moussa, 1986, Un Appel de nuit, Lansman, Belgique, Carnières-Morlanwelz, 1995.

Le Potvin, Sébastien, 2005, Lettre maliennes, Paris, L'Harmattan.

Ly, Ibrahima, 1997, Toiles d'araignées, Arles, Actes Sud, [L'Harmattan, 1982].

Ly, Ibrahima, 1988, Les Noctuelles vivent de larmes, Paris, L'Harmattan.

Ouologuem, Yambo, Le Devoir de violence, Le Serpent à plumes, Paris, 2003 [Seuil, 1968].

Sissoko, Fily Dabo, 1956, La Passion de Djimé, La Tour du guet.

Tapo, Abdoulaye Garbo, 2003, L'Héritage empoisonné, Paris, L'Harmattan.

Traore, Ismailla Samba, 1982, Les Ruchers de la capitale, Paris, L'Harmattan.

Traore, Ismaïla Samba, 2004, Les Amants de l'Esclaverie, Paris, Le Cavalier Bleu.

\section{NOTES}

1. Alpha Oumar KONARE in Bouillon de culture, «spécial Mali », France 2, enregistré en public le $1^{\text {er }}$ décembre 1997

2. Politique Africaine, n $91, \mathrm{p} .17$

3. Xavier Garnier, in Notre Librairie, $\mathrm{n}^{\circ} 148$, Penser la violence, juill-sept 2002, p.54

4. Cf. Sébastien Le Potvin, Lettres maliennes, L'Harmattan, Paris, 2005.

5. Jeune Afrique-L'Intelligent, $n^{\circ} 2327,14$ août $2005: 59$.

6. Gustave-Nicolas Fisher, Psychologie des violences sociales, Dunod, Paris, 2003.

7. Mamadou Bani Diallo, « La femme dans la littérature africaine », Tapama, $\mathrm{n}^{\circ} 3$, déc. 1998, éd. Donniya, Bamako : 42.

8. Les duruni sont des véhicules de transport en commun. Généralement des 404 pick-up bâchées.

9. Souleymane CISSE, Den muso, @ Sisé filimu, 1975, 86 min.

10. Doulaye Konate, Les fondements endogènes d'une culture de paix au Mali, 1999, www.unesco.org/ccp/publications

11. Aminata Dramane Traore, Le viol de l'imaginaire, Actes Sud-Fayard, Paris, 2002, 


\section{RÉSUMÉS}

Dans cet article, une analyse est faite des expressions de la violence dans les œuvres littéraires modernes du Mali. Par le biais de ces ouvrages, on a connaissance d'une forme de perception locale de la violence sociale qui est aussi la perception subjective de l'auteur. Les interprétations de la violence rejoignent celles du peuple qu'elles veulent représenter: les sans-voix, les sans-pouvoir. L'analyse de la littérature moderne malienne montre que les actes de violence domestique accompagnent les mutations d'une société urbaine à la recherche de son identité.

In this article an analysis is made of modern Malian literary works and the appearance of violence in these texts. These novels/books are one way to read about society though a subjective view in which the voice of the author cannot be neglected. Their interpretations merge with the voices of the people they represent or want to represent : those without voices, the powerless. The analysis of modern Malian literature reveals an urban society in search for its identity resulting in acts of domestic violence. 